\title{
Effect of CPAP-therapy on bronchial and nasal inflammation in patients affected by obstructive sleep apnea syndrome*
}

\author{
Donato Lacedonia ${ }^{1}$, Francesco G. Salerno ${ }^{2}$, Giovanna E. Carpagnano ${ }^{1}$, Roberto \\ Sabato $^{1}$, Annarita Depalo ${ }^{1}$, Maria P. Foschino-Barbaro ${ }^{1}$ \\ Institute of Respiratory Diseases, Department of Medical and Occupational Sciences, University of \\ Foggia, Italy \\ 2 Fondazione Salvatore Maugeri, IRCCS, Cassano Murge, Italy
}

SUMMARY

\begin{abstract}
Background: Obstructive sleep apnea syndrome (OSAS) has been shown to be associated to upper and lower airways inflammation. Continuous positive airway pressure (CPAP) is the elective treatment of OSAS. The aim of the present study was to assess the effect of CPAP-therapy on airway and nasal inflammation.

Methods: In 13 non-smoking subjects affected by untreated OSAS and in 11 non-smoking normal volunteers, airway inflammation was detected by analyses of the induced sputum. In the OSAS group measurements were repeated after 1, 10 and 60 days of the appropriate CPAP treatment. In addition, in 12 subjects of the OSAS group, nasal inflammation was detected by the analysis of induced nasal secretions at baseline, and after 1, 10 and 60 days of CPAP treatment.

Results: OSAS patients, compared to normal controls, showed at baseline a higher percentage of neutrophils and a lower percentage of macrophages in the induced sputum. One, 10 and 60 days of appropriate CPAP-therapy did not change the cellular profile of the induced sputum. In addition, in the OSAS patients, the high neutrophilic nasal inflammation present under baseline conditions was not significantly modified by CPAP-therapy. Finally, no patients developed airway hyper-responsiveness after CPAP therapy.

Conclusions: In OSAS subjects, the appropriate CPAP-therapy, while correcting the oxygen desaturation, does not modify the bronchial and nasal inflammatory profile.
\end{abstract}

Keywords: nasal inflammation, neutrophils, CPAP, OSAS

\section{INTRODUCTION}

Obstructive sleep apnea syndrome (OSAS) is characterized by repetitive episodes of upper airway occlusion during sleep. Upper airway inflammation, upper airway mucosal congestion and lower airway inflammation have been shown to be associated with OSAS ${ }^{(1,2)}$. Upper airways, and in particular nasal inflammation, are believed to be the consequence of the mechanical stress associated with the obstruction to the air passage characteristic of the disease ${ }^{(3)}$ or the consequence of repetitive episodes of oxygen desaturation ${ }^{(4)}$.

Olopade and co-workers ${ }^{(5)}$ have shown an increase in exhaled pentane, a marker of airway inflammation, in OSAS subjects in the morning, suggesting the development of airway inflammation during sleep in untreated OSAS patients. Another study by Carpagnano has shown an increase of two markers of inflammation and oxidative stress (IL-6 and 8 -isoprostane) in the breath condensate of OSAS patients, suggesting that inflammation and oxidative stress are characteristic in the airways of OSAS patients ${ }^{(6)}$. Our group has shown that OSAS patients display bronchial inflammation with an increase in the percentage of neutrophils in the induced sputum ${ }^{(7)}$.

Continuous Positive Airways Pressure (CPAP) is the elective treatment of OSAS. Clinical experience shows that CPAP, by increasing the mean pressure in the airways, is capable of maintaining airway patency during sleep in these patients and of correcting the oxygen desaturation (typical of the disease) during sleep.

However, while many studies have demonstrated that the use of CPAP reduces the cardiovascular risk in OSAS patients ${ }^{(8-10)}$, no clear evidence occurs in literature about the effect of CPAP on upper airway inflammation. Skoczy ski et al., have detected an increase of inflammatory cells in nasal lavage after a short use of CPAP ${ }^{(11)}$, while another study showed that the

ABBREVATION LIST: OSAS = Obstructive Sleep Apnea Syndrome; IS = Induced Sputum; NS = Nasal Secretion; AHI = Apnea Hypopnea Index; ODI = Oxygen Desaturation Index; BMI = Body Mass Index; TST90 = Total Sleep Time with $\mathrm{SaO} 2$ under $90 \%$ 
use of CPAP left the inflammatory pattern unchanged ${ }^{(12)}$.

The object of our investigation was to determine the effect of CPAP therapy on airway and nasal inflammation. Airway inflammation was inferred by the cellular analysis of the induced sputum, a technique that is well standardised and widely used for the characterisation of the inflammatory profile in the bronchial compartment in many diseases. Nasal inflammation was detected by the analyses of nasal secretions. Measurements were repeated at various time-points after the onset of the CPAP therapy. Bronchial reactivity was also evaluated by methacholine challenge before and after CPAP.

\section{METHODS}

Study sample

Twenty-five consecutive patients with suspicion of OSAS and 15 age-matched normal volunteers chosen as controls were enrolled. Five patients were not included at visit 1 because no induced sputum was obtained, whereas 3 patients were excluded at visit 4 because they had not used CPAP; 4 patients were excluded because no induced sputum was collected during follow-up, whereas in control group 4 volunteers had no IS. Thus, 13 patients and 11 controls completed the study. All subjects were non-smokers, had no history of asthma, chronic bronchitis, wheezing, allergies or other systemic diseases that might contribute to sleep disorders. All subjects were free from steroids or other anti-inflammatory drugs. None of the subjects were using CPAP therapy. Informed consent was obtained from all the subjects and the Institution Ethic Committee approved the study.

\section{Nocturnal oxymetry}

Nocturnal haemoglobin oxygen saturation was evaluated by finger-pulse oxymetry (Minolta, Pulsox DP 8, Osaka, Japan). A pattern of oxymetry tracing positive for OSAS was defined as a cyclical oxygen desaturation defined as a drop in oxygen saturation $\geq 4 \%$.

\begin{tabular}{lccc}
\hline & OSAS & Normal & p \\
\cline { 2 - 4 } & Mean \pm SD & Mean \pm SD & \\
\hline Subjects (M/F) & $13(11 / 2)$ & $11(6 / 5)$ & \\
Age (year) & $56.08 \pm 9.3$ & $49.55 \pm 7.6$ & $\mathrm{~ns}$ \\
FEV1 (\%) & $95.54 \pm 14.3$ & $111.1 \pm 18.3$ & $<0.05$ \\
FVC (\%) & $96.77 \pm 13.4$ & $113.3 \pm 18.6$ & $<0.05$ \\
Tiff $(\%)$ & $80.21 \pm 0.01$ & $98.45 \pm 0.02$ & $<0.01$ \\
BMI (km/m2) & $38.71 \pm 10$ & $20.43 \pm 2.1$ & $<0.001$ \\
ODI & $51.3 \pm 28.1$ & $1.2 \pm 2.1$ & $<0.0001$ \\
AHI & $48.69 \pm 31$ & & \\
CPAP & $11.53 \pm 2.6$ & & \\
\hline Induced sputum & & & \\
\hline Macrophages & $31.7 \pm 15.4$ & $64.9 \pm 12.8$ & $<0.0001$ \\
Neutrophils & $65.1 \pm 17.2$ & $32 \pm 12.3$ & $<0.0001$ \\
Lymphocytes & $2.6 \pm 3.4$ & $1 \pm 1.3$ & $\mathrm{~ns}$ \\
Eosinophils & $0.38 \pm 0.76$ & $0.1 \pm 0.2$ & $\mathrm{~ns}$ \\
Epithelial Cells & $2.07 \pm 3.2$ & $1.9 \pm 1$ & $\mathrm{~ns}$ \\
\hline
\end{tabular}

Table 1. Demographic and functional characteristics.
Polysomnography

The OSAS subjects were evaluated in the sleep laboratory with an overnight polysomnography (Sleep Lab 1000p, Aequitron Medical, Plymouth, MN, USA) using a standard method during their hospital stay. Sleep stages were scored according to Rechtschaffen and Kales ${ }^{(13)}$. Snoring, sleep position, leg movements and heart rate were recorded. Oro-nasal flow was measured by a nasal canula, whereas abdominal and rib-cage movements were measured by respiratory inductive pletismography and oxyhemoglobin saturation by a finger probe. Sleep-disordered breathing was quantified according to standard criteria ${ }^{(14)}$.

Pulmonary Function Tests and airway responsiveness to methacholine

Pulmonary function tests (Vmax 6200 Autobox System, SensorMedics, Yorba Linda, CA, USA) were done prior to entry into the study to verify that none of the subjects had an obstructive lung disease defined by a FEV1 less than $80 \%$ and a FEV1/FVC ratio lower than 0.7. Airway responsiveness was assessed in all subjects at baseline and, only in OSAS patients, after 1, 10 and 60 days after CPAP use. Bronchial responsiveness was assessed by a standard bronchial provocation test with methacholine $(\mathrm{MCh})$, plotting the percentage drop of FEV1 against the logarithm of the cumulative dose ${ }^{(15)}$. After an initial saline inhalation participants inhaled unbuffered methacholine in doubling doses from $0.03-16$ $\mathrm{mg} / \mathrm{ml}-1$. The MCh aerosol was delivered during quiet breathing by a dosimeter (Mefar, Brescia, Italy). The challenge was stopped if FEV1 decreased $20 \%$ or more from baseline or if the maximal dose of $16 \mathrm{mg} / \mathrm{ml}-1$ was reached. Dose increments were obtained by using MCh concentrations of 1 and $10 \mathrm{mg} / \mathrm{ml}$ with appropriate numbers of inhalations.

\section{Induced sputum and nasal sputum technique}

Inhalation procedure. After baseline $\mathrm{FEV}_{1}$ and $\mathrm{FVC}$ measurements, salbutamol was given by inhalation ( $200 \mu \mathrm{g}$ by MDI) and subjects inhaled hypertonic $(4.5 \%)$ saline nebulized for three periods of 5 minutes each. An ultrasonic nebulizer (DeVilbiss 65, DeVilbiss Corporation, Somerset, PA, USA), nebulized saline solutions. During nebulization the participants had their necks extended to facilitate penetration of the inhaled solution on the whole surface of the nasal mucosa, including the posterior nasal cavity. After 5 minutes of inhalation, the participants were asked to press their left nostril and to blow the right nostril forcefully into a Petri dish. The blowing procedure was repeated for the left nostril ${ }^{(16,17)}$.

Sputum processing. The collected sputum samples and nasal secretion were examined within 2 hrs. Selected portions of the sputum sample originating from the lower respiratory tract were chosen using an inverted microscope, after which they were weighed. Dithiothreitol (DTT, Sputolysin, Calbiochem Corp, San Diego, CA, USA), freshly prepared in a dilution of onein-10 with distilled water, was added in a volume (in $\mu$ l) equal to 4 times the weight of the sputum portion (in $\mathrm{mg}$ ). The selected sputum was placed in a shaking water-bath at $37^{\circ} \mathrm{C}$ for $20 \mathrm{~min}$ and homogenized. It was further diluted with phosphate-buffered saline in a volume equal to the sputum plus DTT. The suspension was 


\begin{tabular}{|c|c|c|c|c|c|}
\hline & Baseline & 1 & 10 & 60 & p \\
\hline \multicolumn{6}{|l|}{ Induced Sputum } \\
\hline Macrophages & $31.7 \pm 15.4$ & $34.7 \pm 15.6$ & $31.7 \pm 15.5$ & $32.9 \pm 19.0$ & ns \\
\hline Neutrophils & $65.1 \pm 17.2$ & $60.8 \pm 15.6$ & $64.4 \pm 15$ & $62.6 \pm 18.5$ & ns \\
\hline Eosinophils & $0.4 \pm 0.8$ & $1.5 \pm 2.0$ & $2.5 \pm 3.0$ & $2.2 \pm 2.2$ & ns \\
\hline Lymphocytes & $2.6 \pm 3.4$ & $1.3 \pm 2.7$ & $0.3 \pm 0.7$ & $1.0 \pm 1.9$ & ns \\
\hline Epithelial cells & $2.1 \pm 3.2$ & $1.4 \pm 3.5$ & $1.0 \pm 2.3$ & $0.8 \pm 1.4$ & ns \\
\hline \multicolumn{6}{|l|}{ Nasal Secretion } \\
\hline Macrophages & $19.1 \pm 19.2$ & $26.3 \pm 5.7$ & $15.1 \pm 11.5$ & $17.1 \pm 8.2$ & ns \\
\hline Neutrophils & $74.6 \pm 25.7$ & $66.6 \pm 32.6$ & $81.6 \pm 14.8$ & $82.2 \pm 8.4$ & ns \\
\hline Eosinophils & $0.3 \pm 0.7$ & $0.5 \pm 0.7$ & $0.2 \pm 0.7$ & $0.1 \pm 0.3$ & ns \\
\hline Lymphocytes & 0 & 0 & 0 & 0 & ns \\
\hline Epithelial cells & $5.3 \pm 9.7$ & $4.7 \pm 9.5$ & $1.8 \pm 3.9$ & $0.6 \pm 1.4$ & ns \\
\hline
\end{tabular}

Table 2. Cells composition in nasal secretion and induced sputum in OSAS patients, at baseline and 1, 10 and 60 days after CPAP.

filtered through gauze to remove any mucus and was centrifuged at $1000 \mathrm{rpm}$ for $5 \mathrm{~min}$. The supernatant was aspirated and frozen at $-70^{\circ} \mathrm{C}$ for later analysis. The cell pellet was resuspended in a volume of PBS equal to that of the sputum plus DTT and PBS, as above. The total cell count (TCC) and viability (Trypan blue exclusion method) were determined using a Burkers chamber haemocytometer. The cell suspension was placed in a Shandon 3 cytocentrifuge (Shandon Southern Instruments, Sewickley, PA, USA) and cytospins were prepared at $450 \mathrm{rpm}$ for $6 \mathrm{~min}$. Cytospin slides were fixed by methanol and stained by May-GrünwaldGiemsa for an overall differential cell count on 500 nucleated non-squamous cells. Only samples with cell viability $>50 \%$ and squamous cell contamination $<20 \%$ were considered adequate. The slides were read by two independent investigators.

\section{Study design}

All subjects underwent the Berlin Questionnaire ${ }^{(18)}$, a pulmonary function test, a nocturnal oxymetry and induced sputum and nasal secretion. All the healthy subjects presented no alterations of nocturnal oxymetry, and were at lower risk of OSAS during the Berlin Questionnaire, whereas only the OSAS subjects underwent a polysomnography. Patients with OSAS who initially accepted CPAP treatment underwent a second sleep study for CPAP manual titration. Optimum CPAP pressure was defined as the pressure value that abolished respiratory events, arousals, and desaturation episodes.

Induced sputum and nasal secretions were collected before and 1,10 and 60 days after titration and were examined in the same manner. The CPAP compliance was estimated by dividing the total recorded hours in the device timer by the number of nights of use between treatment initiation and follow-up examination. All the patients were strongly encouraged to comply with the prescribed therapy. Every patient completed the study and showed a good compliance in CPAP use ( $\geq 4 \mathrm{~h}$ CPAP use/night).

\section{Statistical analysis}

Differences between the two groups were tested by means of the $\mathrm{T}$ test for normally-distributed variables and the Mann-Whitney test for non-normally distributed variables. The changes in airway compositions at different times after CPAP were evaluated by the ANOVA test, in which a $\mathrm{p}$ value $<0.05$ was considered to be statistically significant.

\section{RESULTS}

Subjects

In Table 1 the functional and anthropometrical characteristics of all the subjects are listed; all subjects had a normal pulmonary function test. BMI was significantly higher in OSAS subjects ( $38.7 \pm 10.0$ vs $20.4 \pm 2.0$ ) compared to healthy subjects. AHI in the OSAS group was $49 \pm 31$.

Figure 1 and Table 1 show the cellular profile of the induced sputum in the control and OSAS groups. A significantly higher percentage of neutrophils $(65.2 \pm 17.2$ vs. $32.0 \pm 12.3, \mathrm{p}<0.0001)$, and a significantly lower percentage of macrophages ( $31.7 \pm 15.4$ vs $64.9 \pm 12.8, p<0.0001$ ) were detected in the induced sputum of the OSAS group as compared to the control group. The relative percentages of eosinophils, lymphocytes and epithelial cells were not statistically different between the OSAS and control group.

Analysis of nasal secretions shows the same high percentage of neutrophils in OSAS $(74.6 \pm 25.7)$. The percentage of neutrophils remained high after two months of CPAP use in induced sputum $(65.2 \pm 17.2$ vs $62.7 \pm 18.5, \mathrm{p}=0.96)$ as well as in nasal secretions $(74.6 \pm 25.0$ vs $82.2 \pm 8.4, \mathrm{p}=0.48)$. Besides, no change was observed regarding macrophages, eosinophils and lymphocytes both in induced sputum as in nasal secretions (Table 2, Figure 2).

The Methacholine test performed at baseline was negative (PD20 $>16 \mathrm{mg}$ ) in OSAS patients and in the control group. No patients presented a positive response during and after 2 months of CPAP use.

\section{DISCUSSION}

In the present study, we have shown that patients affected by OSAS display neutrophilic nasal and bronchial inflammation is not corrected by the applied CPAP-therapy.

There is evidence in the literature that patients affected by OSAS develop upper airway inflammation. In patients affected by OSAS, in the nasal mucosa, Rubenstein has shown an increase in the 


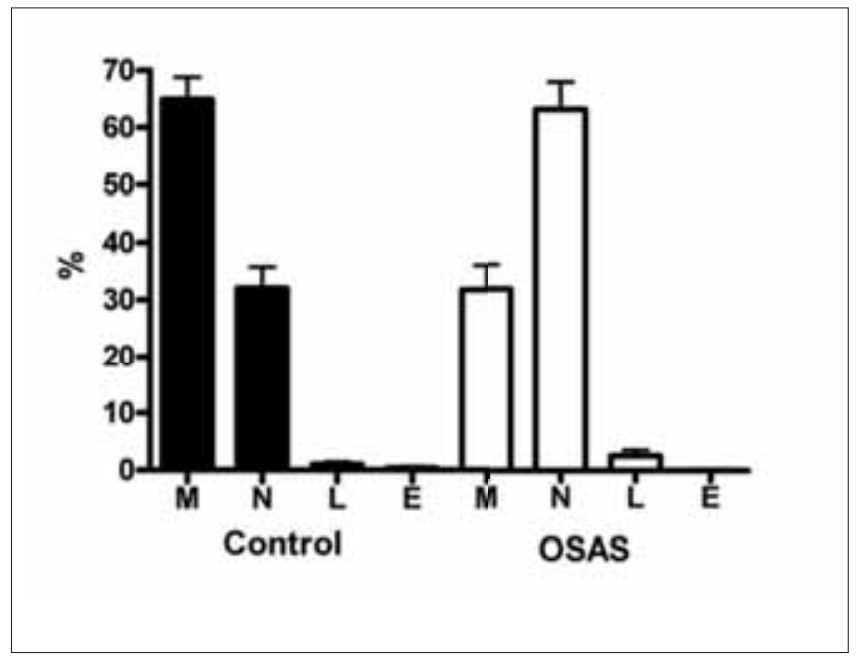

Figure 1. Different percentage of induced sputum cells in OSAS patients and control volunteers.

M: macrophages; N: Neutrophils; L: Lymphocytes; E: Eosinophils

percentage of neutrophils, as well as an increase in other mediators of inflammation such as bradykinin and vasoactive intestinal peptide, suggesting a pathogenic role of nasal inflammation in the upper airway obstruction characteristic of OSAS ${ }^{(1)}$. In addition, Sekosan et al. (2) have shown, in OSAS patients, that the uvula is thicker than normal and that in the lamina propria there is an increase in the number of leukocytes, suggesting again that soft palate inflammation contributes to the upper airway occlusion observed during sleep.

The presence of bronchial inflammation in OSAS has been shown in the literature. In 1997, Olopade (5) showed OSAS patients, in whom pentane and nitric oxide, two markers of airway inflammation, increased after overnight sleep, and an increase of two markers of inflammation and oxidative stress, like IL-6 and 8-isoprostane, in the breath condensate of OSAS patients ${ }^{(6)}$, suggesting that inflammation and oxidative stress are characteristic in the lower airways of these patients. In a previous work, we found an increased percentage of neutrophils in the induced sputum of OSAS patients as compared to normal controls, suggesting the presence of lower airway inflammation (7). However, inflammatory markers do not discriminate between higher and lower airways. Regarding the mechanisms responsible for lower airway inflammation in OSAS subjects there are different possibilities. The mechanical stress exerted on the mucosa of the respiratory system by snoring has been proposed as being responsible for the upper airway inflammation observed in the OSAS population ${ }^{(19)}$. While mechanical stress is still a possible hypothesis, hypoxemia ${ }^{(20,21)}$ and obesity ${ }^{(22,23)}$, alone and in combination, are also theoretical potential causes of bronchial inflammation and their independent role merits further investigation.

Our data show that even if a good compliance and correct use of CPAP can improve sleepiness and reduce cardiovascular risk, they do have adverse effects on local inflammation. The effects of CPAP on systemic inflammations have been extensively examined, but the availability of data about local inflammation is relatively scarce. Nevertheless, it is known that nasal or oral symptoms such as dry nose/mouth and nasal congestion are often present in CPAP-treated patients ${ }^{(24)}$. Moreover, an increase of airway responsiveness was also detected, probably because CPAP treatment is insufficient to achieve suppression of inflammation ${ }^{(12)}$. Indeed, our data, in part, are in accordance with this study. Furthermore, it remains unclear why CPAP therapy doesn't change the nature of local inflammation. At this point we can hypothesize different reasons to explain this effect on local inflammation. The first one could be connected with other pathological conditions commonly present in OSAS patients, such as oesophageal reflux ${ }^{(25)}$, that could be involved in the development of bronchial inflammation. Indeed, by increasing the rate of micro-aspirations, GER could cause a constant noxa on the airway and hence the recruitment of new inflammatory cells in the bronchial tree. Another reason may be a consequence of the mechanical stress derived from the application of a positive pressure inside the airway. Indeed, it's known that the application of CPAP increases leukocyte rolling (reducing velocity) and adhesion in the microvasculature of the upper

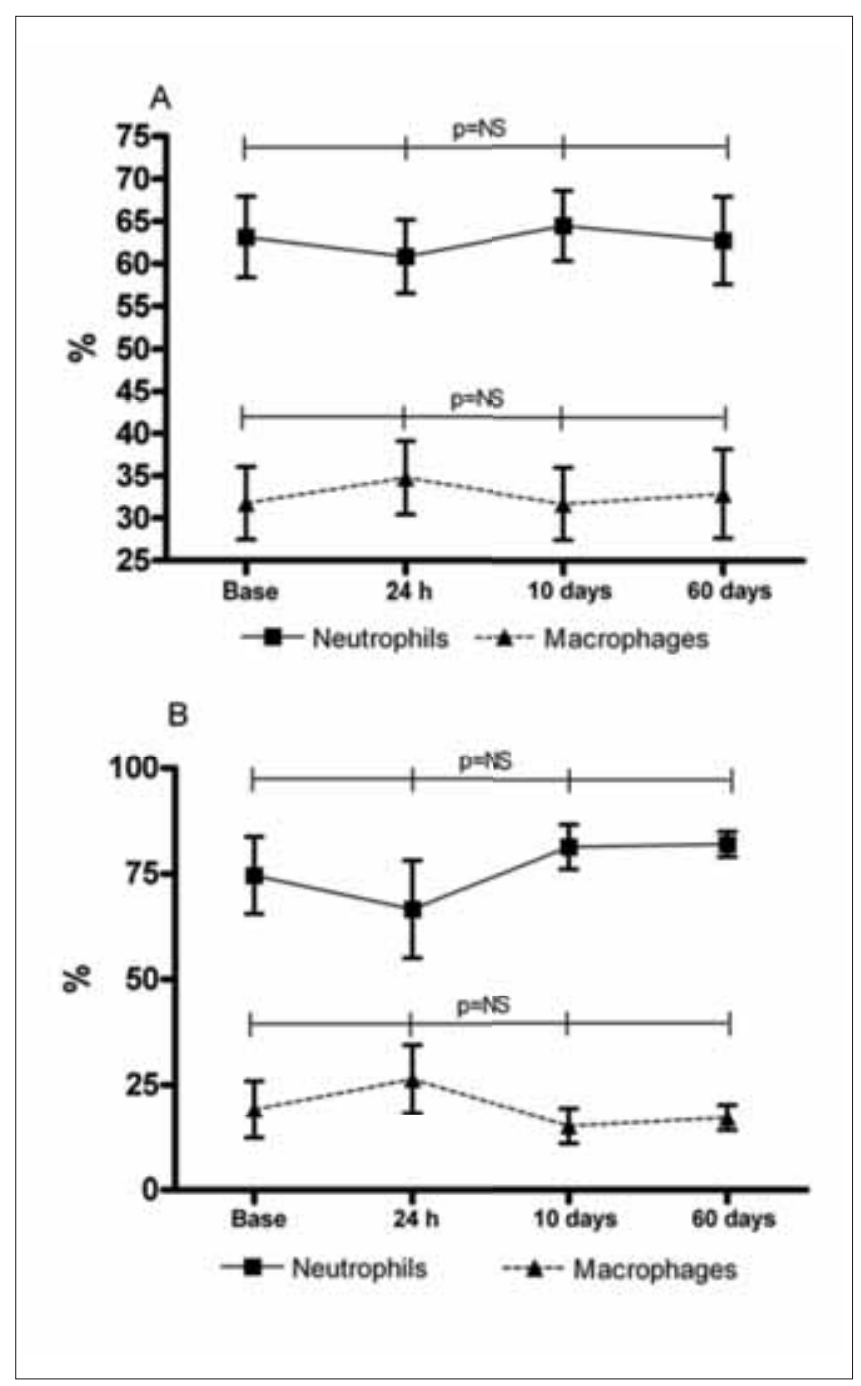

Figure 2. Neutrophils and Macrophages percentage in induced sputum (A) and nasal secretions (B), before and after 1, 10 and 60 days of CPAP therapy. 
airways, accompanied by local leukocyte (neutrophil) infiltration. The extent and intensity of airway distension produce an effect on leukocyte recruitment; this is because the cells that stretch, release various inflammatory mediators, which can lead to leukocyte adhesion ${ }^{(26)}$. This could explain the increase of inflammatory cells, particularly in mucosa of the nose, where there is greater pressure stress. It has been proposed that the increased transpulmonary pressure generated during resistive breathing could cause, at the level of the epithelium or of the respiratory muscles, the increase in cytokines. Indeed, breathing against a resistance (as occurs in sleep apnea) has been shown to increase IL-6 and $\mathrm{TNF}-\alpha$, therefore possibly triggering airway inflammation ${ }^{(27)}$. The explanation for the presence of a bronchial inflammatory process, independent of an underlying chronic bronchitis, is more controversial.

There are reports in the literature on the presence of systemic inflammation in obese patients. Systemic inflammation has been shown to be correlated with high BMI ${ }^{(28)}$, with the inflammation being higher in OSAS patients compared with obese subjects of similar BMI. This systemic inflammation is characterised by high levels of C-reactive protein, IL- 6 , TNF- $\alpha$ ${ }^{(29-31)}$. One of the principal limits of this study is that the control group has a lower BMI than the patients' group; this can be an explanation of the differences in airway inflammation between the two groups. However, it seems that obesity does not have a direct role in airway inflammation, as there are no strict correlations between BMI and neutrophil percentages, in addition to what was previously demonstrated ${ }^{(7,12)}$. Recent reports show that obesity can reduce nasal patency ${ }^{(32)}$ and increase nasal blockage symptoms ${ }^{(33)}$, probably because the fat deposition in the neck and velopharyngeal increase the respiratory effort and stress on upper airway mucosa. In both these studies, OSAS was not excluded, therefore we have no means of knowing whether more blockage symptoms or nasal patency reduction were connected with obesity alone or also with OSAS. It is probable that both conditions play a role in generating an increase of neutrophils in nasal secretion and nasal patency. For this reason, we suppose that the use of CPAP alone cannot reduce local inflammation as long as it is not associated with weight reduction.

On a final note, it should be pointed out that few studies have investigated airway responsiveness in OSAS patients. Thalhofer and co-workers estimated that $4 \%$ of OSAS patients have an increase of bronchial reactivity, and that CPAP therapy worsens bronchial hyper reactivity, but they evaluated only patients that have bronchial hyper reactivity before starting CPAP treatment ${ }^{(34)}$. Other studies ${ }^{(12,35)}$ show that after 1 or 6 weeks of CPAP therapy there was an increase of bronchial reactivity. In the present study, no change arises in airway hyper-responsiveness during and after two months of CPAP treatment. Undoubtedly airway inflammation is associated with bronchial hyper-responsiveness ${ }^{\left({ }^{6}\right)}$. On the other hand, it is possible that the few patients of our population and the absence of changes in the airway cell pattern after treatment could explain the reason for the absence of bronchial reactivity after CPAP. The principal limits of the present study are the relatively low number of patients involved and the BMI difference between the two groups, but this could spur us on to do future research.

In conclusion, it can be said that patients affected by OSAS are seen to display neutrophilic airway inflammation. Even though CPAP therapy improved OSAS symptoms, it probably caused no change in the nasal and airway inflammatory profile or in airway responsiveness. These data suggest that airway inflammation in OSAS is not related only to local mechanical stress or intermitted hypoxia, but could be an epi-phenomenon of a systemic inflammatory status.

\section{FINANCIAL SUPPORT}

All authors disclose no financial relationship with a biotechnology and/or pharmaceutical manufacturer that has an interest in the subject matter or materials discussed in this manuscript.

\section{AUTHOR CONTRIBUTION}

DL designed the study, coordinated it, performed the statistical analysis and wrote the manuscript. FGS designed the study, recruited the patients and assisted in performing the statistical analysis. RS recruited the patients and helped in the coordination of the study. AD carried out the laboratory research and the patients' characterisation for the classification of the different patient groups. GEC participated in the interpretation of the results and critically reviewed the manuscript. MPFB supervised the study as head of the lung research group, participated in its design, coordination and revised the manuscript.

\section{REFERENCES}

1. Rubinstein I. Nasal inflammation is present in patients with obstructive sleep apnea. Laryngoscope. 1995; 105: 175-177.

2. Sekosan M, Zakkar M, Wenig B. Inflammation is present in the uvula mucosa of patients with obstructive sleep apnea. Laryngoscope. 1996; 106: 1018-1020.

3. Boyd JH, Petrof BJ, Hamid Q, Fraser R, Kimoff RJ. Upper airway muscle inflammation and denervation changes in obstructive sleep apnea. Am J Respir Crit Care Med. 2004; 170: 541-546.

4. $\mathrm{Xu} \mathrm{W,} \mathrm{Chi} \mathrm{L,} \mathrm{Row} \mathrm{BW,} \mathrm{et} \mathrm{al.} \mathrm{Increased} \mathrm{oxidative} \mathrm{stress} \mathrm{is}$ associated with chronic intermittent hypoxia-mediated brain cortical neuronal cell apoptosis in a mouse model of sleep apnea. Neuroscience. 2004; 126: 313-323.

5. Olopade CO, Christon JA, Zakkar M, et al. Exhaled pentane and nitric oxide levels in patients with obstructive sleep apnea. Chest. 1997; 111: 1500-1504.

6. Carpagnano GE, Kharitonov SA, Resta O, et al. Increased 8 -isoprostane and interleukin-6 in breath condensate of obstructive sleep apnea patients. Chest. 2002; 122: 1162-1167.

7. Salerno FG, Carpagnano E, Guido P, et al. Airway inflammation in patients affected by obstructive sleep apnea syndrome. Respir Med. 2004; 98: 25-28.

8. Buchner NJ, Sanner BM, Borgel J, Rump LC. Continuous positive airway pressure treatment of mild to moderate obstructive sleep apnea reduces cardiovascular risk. Am J Respir Crit Care Med. 2007; 176: 1274-1280.

9. Robinson GV, Pepperell JC, Segal HC, Davies RJ, Stradling JR. 
Circulating cardiovascular risk factors in obstructive sleep apnoea: data from randomised controlled trial. Thorax. 2004; 59: 777-782.

10. Marin JM, Carrizo SJ, Vicente E, Agusti AG. Long-term cardiovascular outcomes in men with obstructive sleep apnoeahypopnoea with or without treatment with continuous positive airway pressure: an observational study. Lancet. 2005; 365: 10461053.

11. Skoczynski S, Ograbek-Król M, Tazbirek M, Semik-Orzech A, Pierzchała W. Short-term CPAP treatment induces a mild increase in inflammatory cells in patients with sleep apnoea syndrome. Rhinology. 2008; 46: 144-150.

12. Devouassoux G, Lévy P, Rossini E, et al. Sleep apnea is associated with bronchial inflammation and continuous positive airway pressure-induced airway yperresponsiveness J Allergy Clin Immunol. 2007; 119: 597-603

13. Rechtschaffen A, Kales A, editors. Los Angeles: Brain Information Service/Brain Research Institute, University of California; 1968. A manual of standardized terminology, techniques and scoring system of sleep stages in human subjects.

14. The Report of an American Academy of Sleep Medicine Task Force. Sleep-related breathing disorders in adults: recommendations for syndrome definition and measurement techniques in clinical research. Sleep. 1999; 22: 667-689.

15. Crapo RO, Casaburi R, Coates AL, et al. Guidelines for methacholine and exercise challenge testing-1999. This official statement of the American Thoracic Society was adopted by the ATS Board of Directors, July 1999. Am J Respir Crit Care Med. 2000; 161: 309-329.

16. Efthimiadis A, Spanevello A, Hamid Q. Methods of sputum processing for cell counts, immunocytochemistry and in situ hybridisation. Eur Respir J. 2002; 20: 19s-23s

17. Melillo G, Balzano G, Stefanelli et al. Ultrasonic nebulization of hypertonic solution: a new method for obtaining specimens from nasal mucosa for morphologic and biochemical analysis in allergic rhinitis. Allergy. 1998; 53: 794-797.

18. Netzer NC, Stoohs RA, Netzer CM, et al. Using the berlin questionnaire to identify patients at risk for the sleep apnea sindrome. Annals of Internal Medicine. 1999; 131: 485-491.

19. Boyd JH, Petrof BJ, Hamid Q, Fraser R, Kimoff RJ. Upper airway muscle inflammation and denervation changes in obstructive sleep apnea. Am J Respir Crit Care Med. 2004; 170: 541-546.

20. Nacher M, Serrano-Mollar A, Farre R, Panes J, Segui J, Montserrat JM. Recurrent obstructive apneas trigger early systemic inflammation in a rat model of sleep apnea. Respir Physiol Neurobiol. 2007; 155: 93-96.

21. Lévy P, Pépin JL, Arnaud C, et al. Intermittent hypoxia and sleep-disordered breathing: current concepts and perspectives. Eur Respir J. 2008; 32: 1082-1095.

22. Sériès F, Chakir J, Boivin D. Influence of weight and sleep apnea status on immunologic and structural features of the uvula. Am J Respir Crit Care Med. 2004; 170: 1114-1119.

23. Vgontzas AN, Papanicolau DA, Bixler EO, Kales A, Tyson $\mathrm{K}$, Chrousos GP. Elevation of plasma cytokines in disrders of excessive daytime sleepiness: role of sleep disturbance and obesity. Clin Endocrinol Metab. 1997; 82: 1313-1316.

24. Pepin JL, Leger P, Veale D, Langevin B, Robert D, Levy P. Side effects of nasal continuous positive airway pressure in sleep apnea syndrome: study of 193 patients in two French sleep centers.
Chest. 1995; 107: 375-381.

25. Teramoto S, Yamamoto H, Ouchi Y. Gastroesophageal reflux common in patients with sleep apnea rather than snorers without sleep apnea. Chest. 2003; 124: 767-768.

26. Lina H, Lim K, Wagner EM. Airway Distension Promotes Leukocyte Recruitment in Rat Tracheal Circulation. Am J Respir Crit Care Med. 2003; 168: 1068-1074.

27. Vassilakopoulos T, Katsaounou P, Karatza MH, Kollintza A, Zakynthinos S, Roussos C. Strenuous resistive breathing induces plasma cytokines: role of antioxidants and monocytes. Am J Respir Crit Care Med. 2002; 166: 1572-1578.

28. Visser M, Bouter LM, McQuillan GM, Wener MH, Harris TB. Elevated C-reactive protein levels in overweight and obese adults. JAMA. 1999; 282: 2131-2135.

29. Shamsuzzaman AS, Winnicki M, Lanfranchi P, et al. Elevated C-reactive protein in patients with obstructive sleep apnea. Circulation. 2002; 105: 2462-2464.

30. Yokoe T, Minoguchi K, Matsuo H, et al. Elevated levels of C-reactive protein and interleukin-6 in patients with obstructive sleep apnea syndrome are decreased by nasal continuous positive airway pressure. Circulation. 2003; 107: 1129-1134.

31. Minoguchi K, Tazaki T, Yokoe T, et al. Elevated production of tumor necrosis factor- by monocytes in patients with obstructive sleep apnea syndrome. Chest. 2004; 126: 1473-1479.

32. Namysłowski G, Mrówka-Kata K, Scierski W, et al. The nasal airway evaluation in morbid obesity. J physiol Pharmacol. 2005; 56 Suppl 6: 67-75.

33. Johansson L, Bende M. Excessive obesity is related to daily symptoms of nasal blockage. The Skovde population-based study. Rhinology. 2007; 45: 205-207.

34. Thalhofer S, Dorow P, Meissner P, Luding K. Change in bronchial hyperreactivity with nCPAP respiration in patients with sleep related respiratory disorders. Pneumologie. 1997; 51: 767-769.

35. Wenzel G, Schonhofer B, Wenzel M, Kohler D. Bronchial hyperreactivity and nCPAP therapy. Pneumologie. 1997; 51: 770772

36. Huang TJ, Haddad EB, Fox AJ, et al. Contribution of bradykinin $\mathrm{B}(1)$ and $\mathrm{B}(2)$ receptors in allergen-induced bronchial hyperresponsiveness. AmJ Respir Crit Care Med. 1999; 160: 17171723.

Dr Donato Lacedonia Institute of Respiratory Disease Department of Medical and Occupational Sciences University of Foggia Italy

Tel: +39-(0)881-733158

Fax: +39-(0)881-733 040

E-mail: donatolacedonia@gmail. com 